\title{
Steady-State Serum IgG Trough Levels Are Adequate for Pharmacokinetic Assessment in Patients with Immunodeficiencies Receiving Subcutaneous Immune Globulin
}

\author{
Zhaoyang $\mathrm{Li}^{1} \cdot$ Barbara $^{\mathrm{McCoy}^{2}} \cdot$ Werner Engl $^{2} \cdot{\text { Leman } \mathrm{Yel}^{3}}^{3}$
}

Received: 24 September 2020 / Accepted: 4 February 2021 / Published online: 26 May 2021

(C) Ownership of copyright in the Article shall vest in the Rights Holder. When reproducing the Article or extracts from it, the Rights Holder shall acknowledge and reference first publication in the Journal 2021

\begin{abstract}
Patients with primary immunodeficiency diseases often require lifelong immunoglobulin (IG) therapy. Most clinical trials investigating IG therapies characterize serum immunoglobulin $\mathrm{G}(\mathrm{IgG})$ pharmacokinetic (PK) profiles by serially assessing serum IgG levels. This retrospective analysis evaluated whether steady-state serum IgG trough level measurement alone is adequate for PK assessment. Based on individual patient serum IgG trough levels from two pivotal trials (phase 2/3 European [NCT01412385] and North American [NCT01218438]) of weekly 20\% subcutaneous IG (SCIG; Cuvitru, Ig20Gly), trough level-predicted IgG AUC $\left(\mathrm{AUC}_{\tau, \mathrm{tp}}\right)$ were calculated and compared with the reported AUC calculated from serum IgG concentration-time profiles $\left(\mathrm{AUC} \mathrm{C}_{\tau}\right)$. In both studies, mean $\mathrm{AUC}_{\tau, \mathrm{tp}}$ values for Ig20Gly were essentially equivalent to $\mathrm{AUC}_{\tau}$ with point estimates of geometric mean ratio (GMR) of $\mathrm{AUC}_{\tau, \mathrm{tp}} / \mathrm{AUC}_{\tau}$ near 1.0 and $90 \%$ CIs within 0.80-1.25. In contrast, for IVIG, 10\%, mean $\mathrm{AUC}_{\tau, \mathrm{tp}}$ values were lower than $\mathrm{AUC}_{\tau}$ by $>20 \%$, (GMR [90\% CI]: 0.74 [0.70-0.78] and 0.77 [0.73-0.81] for the two studies, respectively). Mean $\mathrm{AUC}_{\tau, \mathrm{tp}}$ values calculated for 4 other SCIG products (based on mean IgG trough levels reported in the literature/labels) were also essentially equivalent to the reported $\mathrm{AUC}_{\tau}$ (differences $<10 \%$ for all except HyQvia, a facilitated SCIG product), while differences for IVIG products were $>20 \%$. In conclusion, steady-state serum IgG levels following weekly SCIG remain stable, allowing for reliable prediction of AUC over the dosing interval using trough IgG levels. These findings indicate that measuring steady-state serum IgG trough levels alone may be adequate for PK assessment of weekly SCIG.
\end{abstract}

Keywords Pharmacokinetics · primary immunodeficiency diseases · subcutaneous immunoglobulin · intravenous immunoglobulin · Cuvitru

\section{Introduction}

Primary immunodeficiency diseases (PID) are a heterogeneous group of $>400$ disorders that are often characterized by absent or deficient antibody production, which can lead to frequent and severe infections $[1,2]$. Patients with PID often require lifelong immunoglobulin (IG) replacement therapy, which

Zhaoyang Li

zhaoyang.li@takeda.com

1 Shire Human Genetic Therapies, Inc., a Takeda company, 650 East Kendall Street, Cambridge, MA 02142, USA

2 Baxalta Innovations $\mathrm{GmbH}$, a Takeda company, Vienna, Austria

3 Baxalta US Inc., a Takeda company, Cambridge, MA, USA reduces the frequency and severity of infections [3, 4]. IG replacement therapy is administered intravenously (IVIG; every 3-4 weeks) or subcutaneously (SCIG; usually daily to every 2 weeks) [5]. Unlike IVIG, SCIG products can be selfadministered at home, are associated with a lower risk of systemic adverse events, and do not require venous access [3-5]. The benefits of SCIG use in patients with immunodeficiencies have led to the development of several SCIG products with various concentrations of immunoglobulin $\mathrm{G}$ (IgG) [1].

The pharmacokinetic (PK) properties of exogenous IG vary by route of administration. While the infusion of IVIG treatment directly into the intravascular space results in an early, high peak of IgG serum concentration $\left(C_{\max }\right)$ followed by distribution and elimination phases, the slower absorption of SCIG from the SC infusion site results in a more gradual and stable increase in $\mathrm{IgG}$ serum concentration for several days, with a lower $C_{\max }$ than the 
peak achieved with IVIG infusions [5-7]. The increased dosing frequency of SCIG compared with IVIG may contribute to the more stable IgG levels, and less fluctuation between peak and trough of steady-state IgG levels throughout the dosing cycle and the occurrence of higher trough IG levels compared with IVIG [6, 8].

Serum IgG trough level monitoring is an important consideration when evaluating IG therapies. While patients show considerable interindividual variability in trough IgG levels, higher serum IgG trough levels (into the normal range for $\mathrm{IgG}$ ) have been associated with a decreased risk of infections and improved clinical outcomes [4, 9]. PK assessments have also been deemed essential by health authorities to support the pharmacological activity and efficacy in the registration of IG products. The US Food and Drug Administration recommends that investigational IG products be evaluated for PK parameters, including the area under the curve (AUC) of serum IgG, for comparisons with effects of previous IVIG treatment $[7,10]$. Dose adjustment of SCIG products may be necessary to achieve an AUC of serum IgG that is equivalent with IVIG products due to the decreased bioavailability of IGadministered SC versus IV [7]. In addition, for new IG products, the European Medicines Agency requires assessment of serum trough levels of IgG as well as other PK parameters in comparison with those achieved with the former IVIG or SCIG product [11].

Clinical investigation studies often use serial blood sampling as a means for understanding PK properties in accordance with regulatory guidance. These studies commonly characterize serum IgG PK profiles through the serial evaluation of serum IgG levels during one or more dosing intervals [12-15]. While serial sampling generally facilitates the provision of valuable PK data, this practice has some drawbacks, including added logistical challenges for the conduct of clinical trials, as well as the burden of multiple blood draws for patients, particularly for pediatric patients, for whom venipuncture can be a highly distressing experience [16]. Blood draws are one of the most common reasons that pediatric patients decline to participate in clinical trials, and the need for additional blood collection outside routine care is a concern expressed by pediatric patients as well as their caregivers [17]. For these patients and others, characterizing PK profiles with fewer blood collection time points, if demonstrated feasible and valid, can be beneficial for eliciting participation from patients in clinical trials and for trial investigators.

Serum IgG generally exhibits prolonged SC absorption, with the time to reach $C_{\max }$ reported in the range of days and long elimination half-life reported in weeks [18]. With weekly dosing for SCIG, peak serum IgG levels at steady state are hardly discernible, and $\operatorname{IgG}$ levels remain rather stable from pre-infusion throughout the treatment interval [12, 14]. Therefore, it is reasonable to believe that the IgG trough level is representative of not only the exposure level at a single time point, but also the total exposure over the dosing interval $\left(\mathrm{AUC}_{\tau}\right)$.

The objective of this retrospective analysis is to evaluate whether serum IgG trough level measurement alone can provide reliable AUC assessment over a dosing interval at steady state compared with that calculated from serial sampling.

\section{Methods}

\section{PK Data Source for Ig20Gly}

Individual patient PK data were obtained from 2 prospective, open-label, noncontrolled multicenter phase $2 / 3$ licensing studies evaluating weekly Ig20Gly (Cuvitru) in patients with PID conducted in Europe (NCT01412385) [12] and North America (NCT01218438) [14]. Inclusion criteria, exclusion criteria, and study designs have previously been described $[12,14]$. Briefly, patients aged $\geq 2$ years with a documented diagnosis of PID requiring IG replacement therapy, for $\geq 3$ months before the first study treatment, and serum IgG trough levels $>5 \mathrm{~g} / \mathrm{L}$ at screening were included.

Patients in the European study received IVIG, $10 \%$ for 13 weeks or SCIG, $16 \%$ for 12 weeks in period 1, and then received weekly SCIG, $20 \%$ at a dose of the period 1 dose adjusted to weekly equivalent for 52 weeks [12]. In the North American study, patients received IVIG, 10\% for 13 weeks in period 1 , and then received weekly SCIG, $20 \%$ at $145 \%$ of the IVIG, $10 \%$ dose adjusted to weekly equivalent for $12-28$ weeks during period 2 and period 3 . This was followed by an individualized dose of SCIG, $20 \%$ for 40 weeks in period 4 [14]. In both trials, $\mathrm{IgG}$ trough levels were assessed in all patients at defined time points throughout the course of each study period, with some additional PK serial sampling collections for patients aged $\geq 12$ years in both of the studies [14]. Abbreviated serial PK sampling was performed for patients aged $<12$ years in the North American study (Supplemental Figure S1).

\section{PK Data Source for Other IG Products}

Mean IgG trough levels were obtained from the published licensing studies for 6 other IG products (SCIG products: Kiovig/GammaGard Liquid [Baxalta US Inc., a member of the Takeda group of companies], HyQvia [Baxalta US Inc., a member of the Takeda group of companies], Gamunex [Grifols Therapeutics Inc.], and Hizentra [Grifols Therapeutics Inc.]; IVIG products: Kiovig/GammaGard and Gamunex). A description of the IG products evaluated is provided in Table 1.

For IVIG and SCIG administration of Kiovig/GammaGard, PK data were obtained from a multicenter, prospective, openlabel North American study of patients with PID [20, 24]. Patients received IV infusions at 3- or 4-week intervals based 
Table 1 Summary of SCIG and IVIG products evaluated

\begin{tabular}{|c|c|c|c|c|}
\hline Product & Manufacturer & Dosage form and strength & $\begin{array}{l}\text { Approved method of } \\
\text { administration and dosage in PID }\end{array}$ & IG content \\
\hline Cuvitru $^{\mathrm{a}}[19]$ & $\begin{array}{l}\text { Baxalta US Inc., a } \\
\text { Takeda company, } \\
\text { Lexington, MA, USA }\end{array}$ & $\begin{array}{l}\text { Immune Globulin Subcutaneous } \\
\text { (Human), 20\% Solution } \\
\text { (200 mg/mL) }\end{array}$ & $\begin{array}{l}\text { SC: } 1.30 \times \text { current } \text { IVIG }^{\text {b }} \text { dose } \\
(\mathrm{g}) / \mathrm{IVIG}^{\mathrm{b}} \text { dose interval (weeks); at } \\
\text { regular intervals from daily to } \\
\text { every } 2 \text { weeks (individualized per } \\
\text { patient). If switching from another } \\
\text { SCIG, administer at same dose as } \\
\text { previous treatment }\end{array}$ & $\begin{array}{l}\geq 98 \% \text { IgG with } \sim 80 \mu \mathrm{g} / \mathrm{mL} \\
\quad \text { IgA }\end{array}$ \\
\hline $\begin{array}{l}\text { Kiovig/GammaGard } \\
\text { Liquid [20] }\end{array}$ & $\begin{array}{l}\text { Baxalta US Inc., a } \\
\text { Takeda company, } \\
\text { Lexington, MA, USA }\end{array}$ & $\begin{array}{l}\text { Immune Globulin Infusion (Human), } \\
10 \% \text { Solution (100 mg/mL) }\end{array}$ & $\begin{array}{l}\text { IV: } 300-600 \mathrm{mg} / \mathrm{kg} \text { every } 3-4 \text { weeks } \\
\text { SC: } 1.37 \times \text { current IV dose }(\mathrm{g}) / \mathrm{IV} \\
\quad \text { dose interval (weeks); weekly }\end{array}$ & $\begin{array}{l}\geq 98 \% \text { IgG with trace } \\
\text { amounts of IgA (average } \\
\text { concentration of } \\
37 \mu \mathrm{g} / \mathrm{mL} \text { ) }\end{array}$ \\
\hline HyQvia/HYQVIA [21] & $\begin{array}{l}\text { Baxalta US Inc., a } \\
\text { Takeda company, } \\
\text { Lexington, MA, USA }\end{array}$ & $\begin{array}{l}\text { Immune Globulin Infusion } 10 \% \\
\text { (Human), Solution }(100 \mathrm{mg} / \mathrm{mL}) \\
\text { and Recombinant Human } \\
\text { Hyaluronidase }(160 \mathrm{U} / \mathrm{mL})\end{array}$ & $\begin{array}{l}\text { SC: } 300-600 \mathrm{mg} / \mathrm{kg} \text { every } 3-4 \text { weeks } \\
\text { for patients naïve to or switching } \\
\text { from another SCIG. If switching } \\
\text { from IVIG, administer at the same } \\
\text { dose and frequency as previous } \\
\text { treatment }\end{array}$ & $\begin{array}{l}\geq 98 \% \text { IgG with trace } \\
\text { amounts of IgA (average } \\
\text { concentration of } \\
37 \mu \mathrm{g} / \mathrm{mL} \text { ) }\end{array}$ \\
\hline Gamunex [22] & $\begin{array}{l}\text { Grifols Therapeutics Inc., } \\
\text { Research Triangle } \\
\text { Park, NC, USA }\end{array}$ & $\begin{array}{c}\text { Immune Globulin Injection } \\
\text { (Human), } 10 \% \text { Solution }\end{array}$ & $\begin{array}{l}\text { IV: } 300-600 \mathrm{mg} / \mathrm{kg} \text { every } 3-4 \text { weeks } \\
\text { SC: } 1.37 \times \text { current IV dose }(\mathrm{g}) / \mathrm{IV} \\
\quad \text { dose interval }(\text { weeks); weekly }\end{array}$ & $\begin{array}{l}\geq 98 \% \text { IgG with trace levels } \\
\quad \text { of fragments, IgA } \\
\text { (average concentration of } \\
\quad 46 \mu \mathrm{g} / \mathrm{mL} \text { ), and IgM }\end{array}$ \\
\hline Hizentra [23] & $\begin{array}{l}\text { CSL Behring AG, Bern, } \\
\text { Switzerland }\end{array}$ & $\begin{array}{l}\text { Immune Globulin (Human), } 20 \% \\
\text { Liquid (200 mg/mL) }\end{array}$ & $\begin{array}{l}\text { SC: } 1.37 \times \text { current IV dose }(\mathrm{g}) / \mathrm{IV} \\
\text { dose interval (weeks); at regular } \\
\text { intervals from daily to every } 2 \\
\text { weeks (individualized per patient) }\end{array}$ & $\begin{array}{l}\geq 98 \% \text { IgG with } \leq 50 \mu \mathrm{g} / \mathrm{mL} \\
\quad \operatorname{IgA}\end{array}$ \\
\hline
\end{tabular}

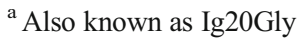

${ }^{\mathrm{b}}$ Or facilitated SCIG

$I G$, immunoglobulin; Ig20Gly, Immune Globulin Subcutaneous (Human), 20\% Solution; IgA, immunoglobulin A; IgG, immunoglobulin G; $I g M$, immunoglobulin $\mathrm{M} ; I V$, intravenous; $I V I G$, intravenous immunoglobulin; $P I D$, primary immunodeficiency diseases; $S C$, subcutaneous; $S C I G$, subcutaneous immunoglobulin

on their pre-study IV dosing regimen during the first study period, and then were switched to receive weekly SC infusions at varying doses (130\% of IV of weekly equivalent for period 2, $137 \%$ of IV of weekly equivalent for period 3 , and an individually adjusted dose for period 4) for the remainder of the study period.

For the facilitated SCIG, HyQvia/HYQVIA (Immune Globulin Infusion, 10\% administered with recombinant human hyaluronidase), PK data were obtained from a prospective, open-label, noncontrolled, multicenter US trial in patients with PID who had received IVIG treatment for $\geq 3$ months, followed by HyQvia/HYQVIA (at $108 \%$ of the weekly equivalent IV dose) administered every 3-4 weeks for approximately 14-18 months [21, 25].

For IVIG and SCIG administrations of Gamunex, PK data were obtained from an open-label, crossover North American trial in patients with PID who had previously received or were currently receiving IG replacement therapy. Patients received Gamunex 200-600 mg/kg IV every 3-4 weeks for at least 3 months, then were switched to weekly SC infusions at a dose adjustment coefficient (DAC) of 1.37 for up to 24 weeks $[22,26]$.

Data for Hizentra were obtained from 2 studies conducted in Europe and the USA. In the European PK substudy, patients with PID who had previously received IVIG treatment (Privigen ${ }^{2}$, Immune Globulin Intravenous [Human], 10\%
Liquid) were switched to weekly subcutaneous (SC) treatment with Hizentra [23, 27]. After a 3-month wash-in/wash-out period, doses were individually adjusted to achieve a systemic serum IgG exposure that was not inferior to that of the previous weekly equivalent IVIG dose. In a US multicenter, prospective, open-label study, patients with PID who had received regular intravenous (IV) treatment with Privigen for $\geq 3$ months prior to enrollment and had achieved serum trough concentration values $\geq 5 \mathrm{~g} / \mathrm{L}$ were switched at study entry to weekly SC treatment with Hizentra at an initial dose calculated using a DAC of 1.3 [28].

\section{Calculation of Trough-Predicted AUC}

Trough-predicted AUC ( $\left.\mathrm{AUC}_{\tau, \mathrm{tp}}\right)$ was calculated based on serum IgG trough levels using the following formula:

$\mathrm{AUC}_{\tau, \mathrm{tp}}=C_{\text {trough,ss }} \times \tau$

where $C_{\text {trough,ss }}$ is the steady-state trough concentration and $\tau$ is the dosing interval.

$\mathrm{AUC}_{\tau, \mathrm{tp}}$ was compared with the reported $\mathrm{AUC}_{\tau}$ that was derived from PK profiles, which were estimated based on serum IgG levels at various time points from serial sampling. For 
Ig20Gly, data for individual patients are available from the two licensing studies. Summary statistics of the AUC values, as well as the geometric mean ratio (GMR) of $\mathrm{AUC}_{\tau, \mathrm{tp}} / \mathrm{AUC}_{\tau}$ (point estimates and $90 \%$ CIs obtained from back-transforming the log-transformed values), were calculated. In addition, BlandAltman plots of log-transformed $\mathrm{AUC}_{\tau, \mathrm{tp}}$ and $\mathrm{AUC}_{\tau}$ values were generated to assess agreement of the methods at individual levels rather than as a summary over the full sample [29].

For other SCIG or IVIG products, the calculation of $\mathrm{AUC}_{\tau, \mathrm{tp}}$ was based on the published mean serum trough data, and the $\%$ difference between $\mathrm{AUC}_{\tau, \mathrm{tp}}$ and $\mathrm{AUC}_{\tau}$ was determined using the following formula:

$\%$ difference $=\left[\left(\mathrm{AUC}_{\tau, \mathrm{tp}}-\mathrm{AUC}_{\tau}\right) / \mathrm{AUC}_{\tau}\right] \times 100$

For completeness, for Ig20Gly, the mean trough data were also used to calculate $\mathrm{AUC}_{\tau, \mathrm{tp}}$ and the \% difference between $\mathrm{AUC}_{\tau, \mathrm{tp}}$ and $\mathrm{AUC}_{\tau}$.

For all studies included in this retrospective analysis, patients or their guardians provided written informed consent according to local consent procedures in accordance with the ethical standards of their respective institutional research committees and with the 1964 Helsinki declaration and its later amendments [12, 14, 24-28].

\section{Results}

In the two Ig20Gly licensing studies, mean values of $\mathrm{AUC}_{\tau, \mathrm{tp}}$ for SCIG, $16 \%$ and SCIG, $20 \%$ were essentially equivalent to the reported $\mathrm{AUC}_{\tau}$, with point estimates of GMR of $\mathrm{AUC}_{\tau, \mathrm{tp}}$ versus $\mathrm{AUC}_{\tau}$ between 0.98 and 1.09, and all $90 \%$ CIs within the commonly used equivalence limit of $0.80-1.25$ (Fig. 1, Supplemental Table S1). By comparison, for IVIG, mean values of $\mathrm{AUC}_{\tau, \mathrm{tp}}$ in these 2 studies were consistently lower than the reported $\mathrm{AUC}_{\tau}$ by greater than $20 \%$; the point estimate of GMR $(90 \% \mathrm{CI})$ of $\mathrm{AUC}_{\tau, \mathrm{tp}}$ versus $\mathrm{AUC}_{\tau}$ was 0.74 $(0.70-0.78)$ and $0.77(0.73-0.81)$ for the European study and North American study, respectively (Fig. 1, Supplemental Table S1). Bland-Altman plots for IVIG, $10 \%$ (Fig. 2a) and SCIG treatment (SCIG, 16\% and SCIG, 20\%) (Fig. 2b) administration show the individual agreement for $\mathrm{AUC}_{\tau, \mathrm{tp}}$ and $\mathrm{AUC}_{\tau}$ with back-transformed values to account for the approximately Gaussian distribution of the AUCs. Agreement was lower for IVIG, $10 \%$ than for SCIG treatment.

$\mathrm{AUC}_{\tau, \mathrm{tp}}$ were also calculated for other SCIG and IVIG products, based on mean serum IgG trough levels in the published licensing studies (Table 2). Differences between the calculated $\mathrm{AUC}_{\tau, \mathrm{tp}}$ and reported $\mathrm{AUC}_{\tau}$ were $>20 \%$ for both IVIG therapies, while for the SCIG therapies, $\mathrm{AUC}_{\tau, \mathrm{tp}}$ and $\mathrm{AUC}_{\tau}$ were all within $\pm 10 \%$ of each other, except for HyQvia. The mean $C_{\max }$ was more than double the $C_{\text {trough }}$ for all IVIG products, while differences between the means of $C_{\max }$ and $C_{\text {trough }}$ for SCIG products were in the range of 1.9 to $23.7 \%$ (mostly within $15 \%$ ), except for HyQvia (33.0\%). For the 2 products administered as both SCIG and IVIG therapy (Kiovig/GammaGard and Gamunex), the IV products resulted in lower mean values of $\mathrm{AUC}_{\tau, \mathrm{p}}$ and a greater difference between $\mathrm{AUC}_{\tau, \mathrm{tp}}$ and $\mathrm{AUC}_{\tau}$ compared with the corresponding $\mathrm{SC}$ products, and greater mean $C_{\max }$ values and lower mean $C_{\text {trough }}$ values than the SC products.

\section{Discussion}

This retrospective analysis sought to determine whether measurement of serum IgG trough levels alone is sufficient for steady-state PK assessment of SCIG therapies in patients who have PID. Given the PK characteristics of serum IgG after SC administration, i.e., long elimination half-life (in weeks) and prolonged SC absorption (in days), it is expected that the serum levels of IgG over a 1-week dosing interval at steady state would remain stable, with essentially no discernible peak in serum IgG levels. This is demonstrated by the data for Ig20Gly $[12,14]$ and other conventional SCIG products where the means of $C_{\max }$ and $C_{\text {trough }}$ for SCIG products were in the range of 1.9 to $23.7 \%$ (mostly within $15 \%$ ) of each other. By comparison, the mean $C_{\max }$ was more than double the $C_{\text {trough }}$ for all IVIG products. This minimal fluctuation between peak and trough concentrations after SC administration of a SCIG therapy at steady state has formed the foundation of this proposed trough-based PK assessment.

$\mathrm{AUC}_{\tau, \mathrm{tp}}$ calculated based on steady-state trough levels for Ig20Gly administered weekly was found to be equivalent to the $\mathrm{AUC}_{\tau}$ reported in pivotal studies using a serial sampling method $[12,14]$. The agreement between $\mathrm{AUC}_{\tau, \mathrm{tp}}$ and $\mathrm{AUC}_{\tau}$ for Ig20Gly treatment was found to be as expected given a generally reported range of $15-20 \%$ for the coefficient of variation of IgG assays. Similarly, for other SCIG products, when $\mathrm{AUC}_{\tau, \mathrm{tp}}$ was derived from mean serum trough IgG levels, it was within $\pm 10 \%$ of the reported $\mathrm{AUC}_{\tau}$ for each individual product. In contrast, the $\mathrm{AUC}_{\tau, \text { tp }}$ for IVIG and facilitated SCIG products was considerably lower than the reported $\mathrm{AUC}_{\tau}$, and the agreement between $\mathrm{AUC}_{\tau, \mathrm{tp}}$ and $\mathrm{AUC}_{\tau}$ was much lower for IVIG, $10 \%$. These findings suggest that for SCIG therapies, steady-state serum IgG trough levels are representative of not only the exposure level at a single time point but also the total exposure over the dosing interval $\left(\mathrm{AUC}_{\tau}\right)$; therefore, the measurement of serum trough level alone at steady state may be adequate for PK assessment of weekly conventional SCIG treatment in patients with PID.

HyQvia/HYQVIA is a facilitated SCIG product, which is co-administered with recombinant human hyaluronidase, an absorption enhancer [21]. It is likely that the increased 


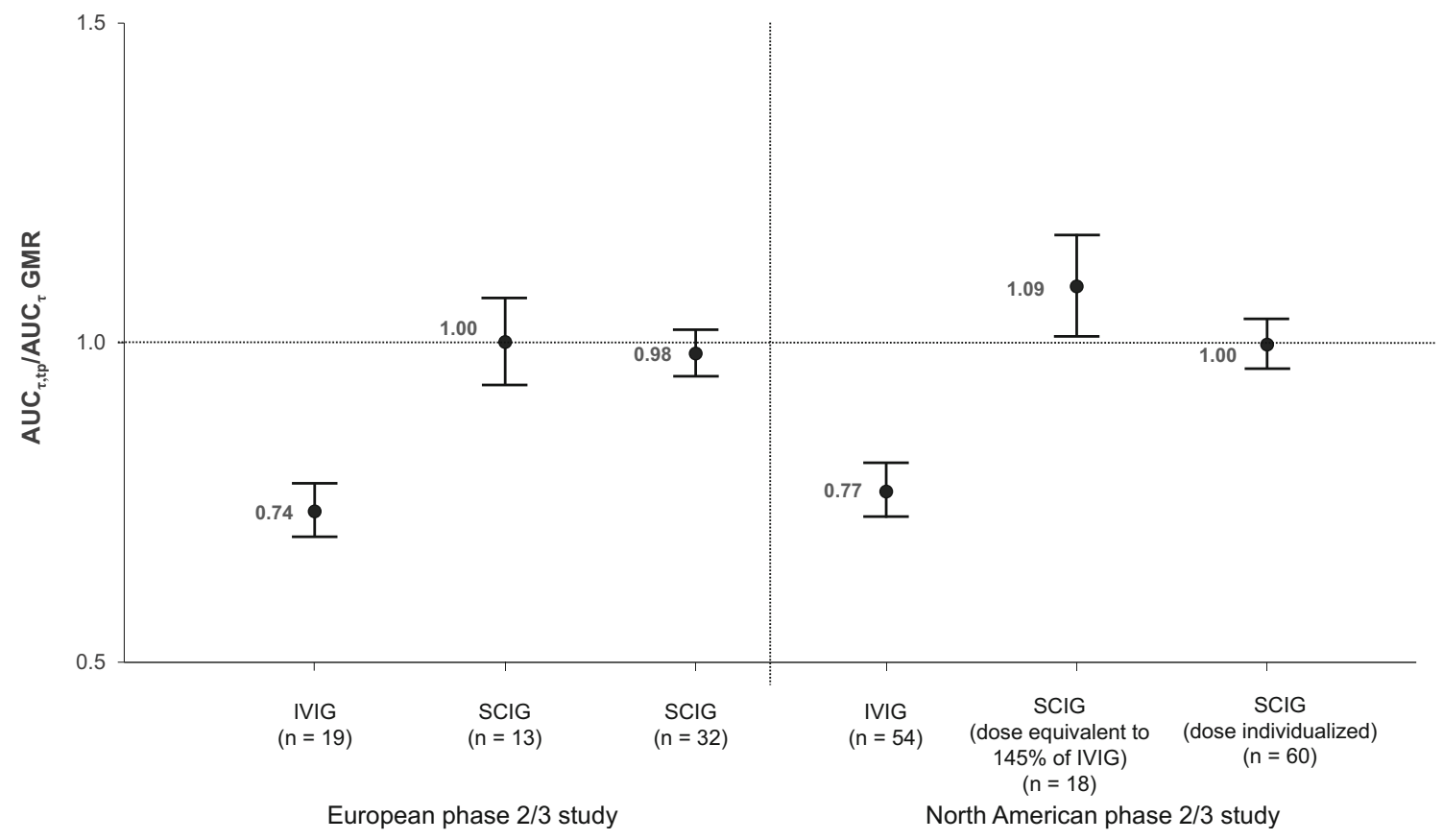

Fig. 1 Trough-predicted $\mathrm{AUC}_{\tau, \mathrm{tp}}$ versus reported $\mathrm{AUC}_{\tau}$ (90\% CIs) for IVIG and SCIG in two phase 2/3 licensing studies of Ig20Gly (Cuvitru), calculated using individual patient data.

Error bars represent $90 \%$ CIs. Horizontal reference line $=$ GMR of 1.0 . $\mathrm{AUC}_{\tau}$, area under the curve calculated from serum IgG concentrationtime profiles over a dosing interval; $\mathrm{AUC}_{\tau, \mathrm{tp}}$, trough level-predicted area under the curve over a dosing interval; CI, confidence interval;

absorption rate of serum $\operatorname{IgG}$ associated with this treatment together with the dosing schedule of 3-4 weeks would result in higher $C_{\max }$ and a consequently larger difference between $C_{\max }$ and $C_{\text {trough }}$, and therefore contributed to our findings for this product that trough-predicted $\mathrm{AUC}_{\tau, \mathrm{p}}$ is not equivalent to the reported $\mathrm{AUC}_{\tau}$. Similarly, the larger difference between $\mathrm{AUC}_{\tau, \mathrm{tp}}$ and the reported $\mathrm{AUC}_{\tau}$ observed for IVIG products is likely the result of their increased bioavailability relative to

GMR, geometric mean ratio; Ig20Gly, Immune Globulin Subcutaneous (Human) 20\% Solution; IVIG, intravenous immunoglobulin; SCIG, subcutaneous immunoglobulin; $\tau$, dosing interval (3-4 weeks for IVIG [Kiovig/GammaGard and Gamunex] and facilitated SCIG [HyQvia/HYQVIA], and 1 week for SCIG [Cuvitru, Kiovig/ GammaGard, Gamunex, and Hizentra])

SCIG products, increasing $C_{\max }$, and the longer dosing interval, which allows for more IgG to be metabolized before the next dose is administered, lowering $C_{\min }$ [5-8]. Given the larger variation in serum IgG levels from peak to trough during the 3-4-week dosing interval for HyQvia/HYQVIA and across IVIG products, IgG trough levels alone are not sufficient for PK assessment at steady state in patients receiving IVIG or HyQvia/HYQVIA.

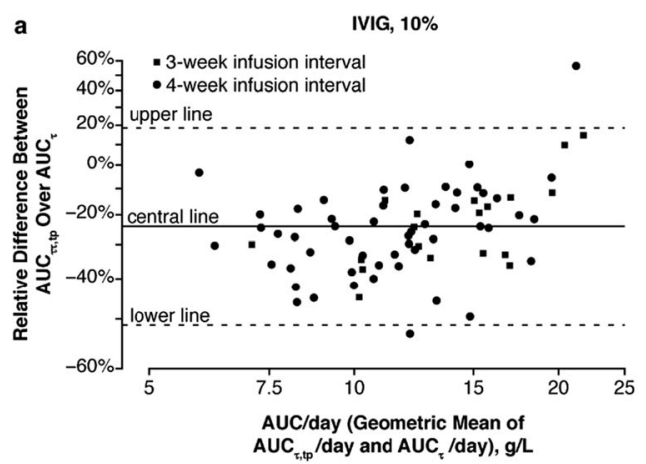

Fig. 2 Agreement between trough-predicted $\mathrm{AUC}_{\tau, \mathrm{t}}$ versus reported $\mathrm{AUC}_{\tau}$ for IVIG (a) and SCIG (b) in two phase 2/3 licensing studies of Ig20Gly. To facilitate the interpretation, back-transformed values are shown. The central (upper/lower) line represents the mean $( \pm 1.96$ standard deviations) of the log-transformed difference of predicted vs reported values to account for the approximately Gaussian distribution of the

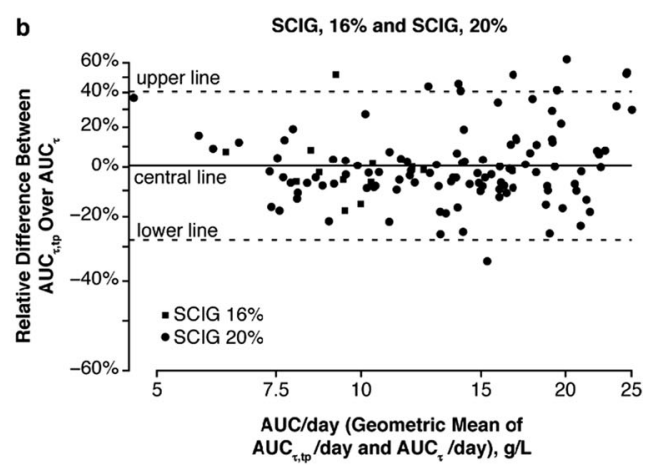

AUCs. $\mathrm{AUC}_{\tau}$, area under the curve calculated from serum IgG concentration-time profiles over a dosing interval.

$\mathrm{AUC}_{\tau, \mathrm{tp}}$, trough level-predicted area under the curve over a dosing interval; Ig20Gly, Immune Globulin Subcutaneous (Human) 20\% Solution; IVIG, intravenous immunoglobulin; SCIG, subcutaneous immunoglobulin; $\tau$, dosing interval (3-4 weeks for IVIG, $10 \%$, and 1 week for SCIG, $16 \%$ and SCIG, $20 \%$ ) 


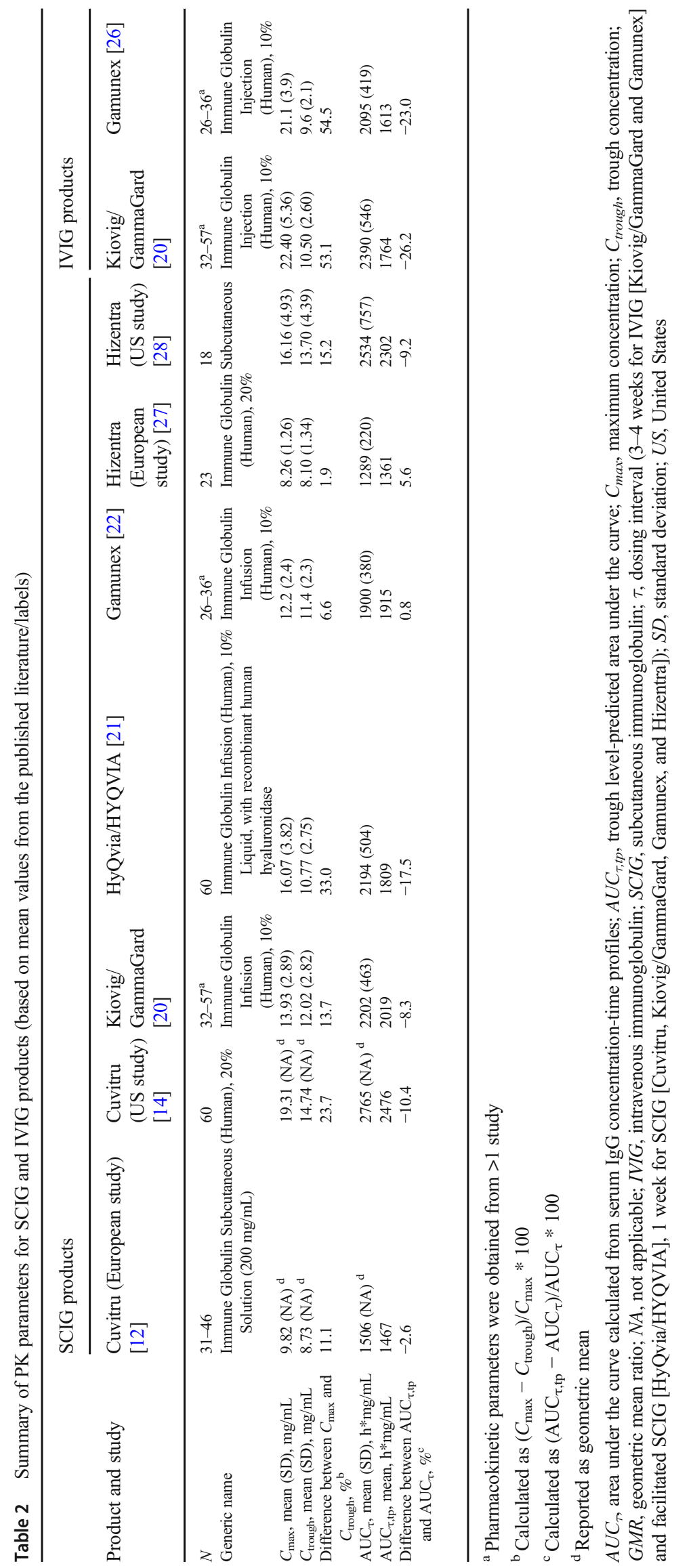


Despite the fact that other PK parameters, such as volume of distribution, elimination half-life, and clearance, are occasionally reported for SCIG, these parameters are not truly accurately estimated from a steady-state PK profile over a 1 -week dosing interval due to the prolonged absorption and long elimination half-life. In the authors' opinion, the systemic exposure parameters, including $C_{\text {trough }}$ and $\mathrm{AUC}_{\tau}$, are the most relevant PK assessment at steady state for SCIG products, and our proposed serum trough alone approach will cover both the single-point exposure and the total exposure. Therefore, the value of characterizing PK profiles of serum IgG over the 1week dosing interval by serial sampling is very limited.

One limitation of this analysis is that because this was a retrospective analysis, direct access to individual patient data was not available for all products; in these cases, the analysis was conducted only on the mean values reported in the published literature, and a Bland-Altman plot could not be provided. In addition, conclusions about the value of measuring serum IgG trough levels alone for PK assessment of SCIG products at steady state are limited in this manuscript to SCIG treatment received on a weekly basis, regardless of the dose level (100-145\% of the equivalent prior IVIG dose in the Ig20Gly studies).

In conclusion, steady-state serum IgG levels remain stable following weekly SCIG treatment in patients with PID, enabling reliable prediction of total exposure $\left(\mathrm{AUC}_{\tau}\right)$ using serum IgG trough levels alone. Our findings appear to be generalizable across all conventional SCIG products. These results indicate that measuring steady-state serum $\operatorname{IgG}$ trough levels alone for PK assessment of weekly SCIG treatment is a reasonable and beneficial alternative to serial PK sampling during clinical development and beyond. For patients and investigators, the use of steady-state IgG trough levels for PK assessment offers multiple benefits, including more efficient clinical trial conduct through decreases in study costs and logistical complexity and, more importantly, a reduction in the patient burden of frequent blood sampling.

Supplementary Information The online version contains supplementary material available at https://doi.org/10.1007/s10875-021-00990-z.

Acknowledgements The authors thank the investigators who conducted the clinical trials that were referenced, the patients who participated, and any caregivers and study-site personnel. Medical writing support was provided by Amanda Borrow, PhD, of Oxford PharmaGenesis Inc., and funded by Takeda Development Center Americas, Inc. This study was funded by Baxalta US Inc., a member of the Takeda group of companies.

Author Contribution All authors contributed to the writing of this manuscript, approved the final version for submission, and are accountable for all aspects of the work. Zhaoyang Li conceived, designed, and performed the data analysis and data interpretation of the manuscript. Barbara McCoy contributed to data interpretation of the manuscript. Werner Engl contributed the Bland-Altman plot and to data interpretation of the manuscript. Leman Yel contributed to data collection, design of data analysis, and data interpretation of the manuscript.

Funding This study was funded by Baxalta US Inc., Bannockburn, IL, a member of the Takeda group of companies.

Availability of Data and Materials The datasets, including the redacted study protocol, redacted statistical analysis plan, and individual participants data supporting the results reported in this article, will be made available within 3 months from initial request, to researchers who provide a methodologically sound proposal. The data will be provided after its deidentification, in compliance with applicable privacy laws, data protection, and requirements for consent and anonymization.

\section{Declarations}

Ethics Approval and Consent to Participate The clinical studies of which the primary results were reported previously were performed in accordance with the Declaration of Helsinki and the international standards of Good Clinical Practice as well as in accordance with the ethical standards of the institutional and/or national research committee. For all of the included studies, written informed consent was obtained from all patients or their legally authorized representatives. Due to the retrospective and de-identified nature of this analysis, individualized patient consent was not obtained.

\section{Consent for Publication Not applicable.}

Competing Interests Zhaoyang Li, Barbara McCoy, Werner Engl, and Leman Yel are employees and shareholders of the Takeda group of companies.

Open Access This article is licensed under a Creative Commons Attribution 4.0 International License, which permits use, sharing, adaptation, distribution and reproduction in any medium or format, as long as you give appropriate credit to the original author(s) and the source, provide a link to the Creative Commons licence, and indicate if changes were made. The images or other third party material in this article are included in the article's Creative Commons licence, unless indicated otherwise in a credit line to the material. If material is not included in the article's Creative Commons licence and your intended use is not permitted by statutory regulation or exceeds the permitted use, you will need to obtain permission directly from the copyright holder. To view a copy of this licence, visit http://creativecommons.org/licenses/by/4.0/.

\section{References}

1. Kobrynski L. Subcutaneous immunoglobulin therapy: a new option for patients with primary immunodeficiency diseases. Biologics. 2012;6:277-87.

2. Tangye SG, Al-Herz W, Bousfiha A, Chatila T, CunninghamRundles C, Etzioni A, et al. Human inborn errors of immunity: 2019 update on the classification from the International Union of Immunological Societies Expert Committee. J Clin Immunol. 2020;40:24-64.

3. Perez EE, Orange JS, Bonilla F, Chinen J, Chinn IK, Dorsey M, et al. Update on the use of immunoglobulin in human disease: a review of evidence. J Allergy Clin Immunol. 2017;139:S1-46. 
4. Shehata N, Palda V, Bowen T, Haddad E, Issekutz TB, Mazer B, et al. The use of immunoglobulin therapy for patients with primary immune deficiency: an evidence-based practice guideline. Transfus Med Rev. 2010;24(Suppl 1):S28-50.

5. Wasserman RL. Progress in gammaglobulin therapy for immunodeficiency: from subcutaneous to intravenous infusions and back again. J Clin Immunol. 2012;32:1153-64.

6. Berger M, Rojavin M, Kiessling P, Zenker O. Pharmacokinetics of subcutaneous immunoglobulin and their use in dosing of replacement therapy in patients with primary immunodeficiencies. Clin Immunol. 2011;139:133-41.

7. Berger M, Jolles S, Orange JS, Sleasman JW. Bioavailability of IgG administered by the subcutaneous route. J Clin Immunol. 2013;33: 984-90.

8. Berger M. Choices in IgG replacement therapy for primary immune deficiency diseases: subcutaneous IgG vs. intravenous $\operatorname{IgG}$ and selecting an optimal dose. Curr Opin Allergy Clin Immunol. 2011;11:532-8.

9. Orange JS, Grossman WJ, Navickis RJ, Wilkes MM. Impact of trough IgG on pneumonia incidence in primary immunodeficiency: a meta-analysis of clinical studies. Clin Immunol. 2010;137:21-30.

10. U.S. Department of Health and Human Services, Food and Drug Administration, Center for Biologics Evaluation and Research. Guidance for industry: safety, efficacy, and pharmacokinetic studies to support marketing of immune globulin intravenous (human) as replacement therapy for primary humoral immunodeficiency. 2008. https://www.fda.gov/media/124333/download. Accessed 1 Jul 2020.

11. European Medicines Agency. Guideline on the clinical investigation of human normal immunoglobulin for subcutaneous and/or intramuscular administration (SCIg/IMIg) (EMA/CHMP/BPWP/ 410415/2011 rev 1). 2015. https://www.ema.europa.eu/en/ documents/scientific-guideline/guideline-clinical-investigationhuman-normal-immunoglobulin-subcutaneous/intramuscularadministration-scig/imig_en.pdf. Accessed 1 Jul 2020.

12. Borte M, Kriván G, Derfalvi B, Maródi L, Harrer T, Jolles S, et al. Efficacy, safety, tolerability and pharmacokinetics of a novel human immune globulin subcutaneous, $20 \%$ : a phase $2 / 3$ study in Europe in patients with primary immunodeficiencies. Clin Exp Immunol. 2017;187:146-59.

13. Kobayashi RH, Gupta S, Melamed I, Mandujano JF, Kobayashi AL, Ritchie B, et al. Clinical efficacy, safety and tolerability of a new subcutaneous immunoglobulin 16.5\% (Octanorm [Cutaquigß]) in the treatment of patients with primary immunodeficiencies. Front Immunol. 2019;10:40.

14. Suez D, Stein M, Gupta S, Hussain I, Melamed I, Paris K, et al. Efficacy, safety, and pharmacokinetics of a novel human immune globulin subcutaneous, $20 \%$ in patients with primary immunodeficiency diseases in North America. J Clin Immunol. 2016;36:70012.

15. Wasserman RL, Melamed IR, Stein MR, Jolles S, Norton M, Moy $\mathrm{JN}$, et al. Evaluation of the safety, tolerability, and pharmacokinetics of Gammaplex® $10 \%$ versus Gammaplex ${ }^{\circledR} 5 \%$ in subjects with primary immunodeficiency. J Clin Immunol. 2017;37:301-10.

16. Humphrey GB, Boon CM. van Linden van den Heuvell GF, van de Wiel HB. The occurrence of high levels of acute behavioral distress in children and adolescents undergoing routine venipunctures. Pediatrics. 1992;90:87-91.

17. Shilling V, Williamson PR, Hickey H, Sowden E, Smyth RL, Young B. Processes in recruitment to randomised controlled trials of medicines for children (RECRUIT): a qualitative study. Health Technol Assess. 2011;15:1-116.

18. Gustafson R, Gardulf A, Hansen S, Leibl H, Engl W, Lindén M, et al. Rapid subcutaneous immunoglobulin administration every second week results in high and stable serum immunoglobulin G levels in patients with primary antibody deficiencies. Clin Exp Immunol. 2008;152:274-9.

19. CUVITRU, Immune Globulin Subcutaneous (Human), 20\% solution [prescribing information]. Lexington, MA: Baxalta US Inc.; 2019.

20. GAMMAGARD LIQUID, Immune Globulin Infusion (Human), $10 \%$ solution [prescribing information]. Westlake Village, CA: Baxalta US Inc.; 2016.

21. HYQVIA, Immune Globulin Infusion $10 \%$ (Human) with recombinant human hyaluronidase [prescribing information]. Lexington, MA: Baxalta US Inc.; 2020.

22. GAMUNEX®-C, Immune Globulin Injection (Human). 10\% caprylate/chromatography purified [prescribing information]. Research Triangle Park: Grifols Therapeutics LLC; 2020.

23. HIZENTRA, Immune Globulin Subcutaneous (Human), 20\% liquid [prescribing information]. Bern. CSL Behring AG: Switzerland; 2020.

24. Wasserman RL, Melamed I, Kobrynski L, Strausbaugh SD, Stein MR, Sharkhawy M, et al. Efficacy, safety, and pharmacokinetics of a 10\% liquid immune globulin preparation (GAMMAGARD LIQUID, 10\%) administered subcutaneously in subjects with primary immunodeficiency disease. J Clin Immunol. 2011;31:323-31.

25. Wasserman RL, Melamed I, Stein MR, Gupta S, Puck J, Engl W, et al. Recombinant human hyaluronidase-facilitated subcutaneous infusion of human immunoglobulins for primary immunodeficiency. J Allergy Clin Immunol. 2012;130:951-7.

26. Wasserman RL, Irani AM, Tracy J, Tsoukas C, Stark D, Levy R, et al. Pharmacokinetics and safety of subcutaneous immune globulin (human), 10\% caprylate/chromatography purified in patients with primary immunodeficiency disease. Clin Exp Immunol. 2010;161:518-26.

27. Jolles S, Bernatowska E, de Gracia J, Borte M, Cristea V, Peter HH, et al. Efficacy and safety of Hizentra ${ }^{\circledR}$ in patients with primary immunodeficiency after a dose-equivalent switch from intravenous or subcutaneous replacement therapy. Clin Immunol. 2011;141:90 102.

28. Wasserman RL, Melamed I, Nelson RP Jr, Knutsen AP, Fasano MB, Stein MR, et al. Pharmacokinetics of subcutaneous IgPro20 in patients with primary immunodeficiency. Clin Pharmacokinet. 2011;50:405-14.

29. Bland JM, Altman DG. Statistical methods for assessing agreement between two methods of clinical measurement. Lancet. 1986;1: 307-10.

Publisher's Note Springer Nature remains neutral with regard to jurisdictional claims in published maps and institutional affiliations. 